\title{
Introduction: modern benchmark analysis for environmental risk assessment
}

\author{
R. Webster West • Walter W. Piegorsch
}

Published online: 28 February 2008

(C) Springer Science+Business Media, LLC 2008

This special issue of Environmental and Ecological Statistics is dedicated to the statistical issues involved in benchmark dose analysis. First proposed by Crump (1984) as an alternative to the much-maligned NOAEL (no-observed-adverse-effect level) method for identifying safe or acceptable exposure levels of toxic compounds, the development of benchmark methodology has been an active research area in risk assessment for more than two decades; the approach is now increasingly used for regulatory purposes (U.S. EPA 2000). In benchmark analysis, one characterizes exposure risk as the probability of an adverse effect, and relates this to the exposure level or dosage of a particular hazardous agent. Statistical complexity arises when constructing point and interval estimates for the risk-or functions of it - and for the dose level at which a specific "benchmark risk" is attained. This latter quantity is called a "benchmark dose," or BMD, and it is a key output value in risk-analytic benchmark computations. From this simple idea, there have merged a number of outstanding statistical issues for applying the methodology, in a variety of dose-response settings. Many of these relate to use of the original derivation of BMD as a replacement for the NOAEL, as expertly discussed by Ralph Kodell in the first article of this special issue. BMD originator Kenny Crump and co-author Justin Teeguarden follow with an article highlighting the issues involved when applying the benchmark dose approach to summary dose-response data. Crump and Teeguarden give a new, hybrid Monte Carlo method for performing BMD estimation under this increasingly common paradigm.

\footnotetext{
R. W. West ( $\square)$

Department of Statistics, 3143 TAMU, Texas A\&M University, College Station, TX 77843, USA

e-mail: websterwest@yahoo.com

W. W. Piegorsch

Department of Mathematics and BIO5 Institute, University of Arizona, Tucson, AZ 85721, USA

e-mail: piegorsc@math.sc.edu
} 
The next two articles in this special issue deal with the connection between BMD estimation and dose-response modeling. Mehdi Razzaghi introduces the beta-normal distribution for benchmark analysis with dose-response models under quantitative (continuous) responses. Matthew Wheeler and John Bailer follow with a discussion of the model averaging approach for dealing with dose-response model uncertainty, a growing concern in BMD estimation. The final two articles deal with the specific question of finding lower confidence limits on BMDs at pre-specified benchmark risks. Buckley et al. evaluate different confidence limit procedures for a simple one-hit model historically popular for benchmark modeling. Along with co-author Daniela Nitcheva, we then end with a focus on how the bootstrap approach can improve construction of simultaneous inferences for BMDs, centering attention on a two-stage version of Armitage and Doll's (1954) famous multistage model.

Taken together, this collection of articles serves as an informative and useful survey of current research in benchmark analysis. The papers characterize the many different ways Crump's original BMD concept has been applied in quantitative risk analysis, and the interesting statistical questions that have evolved as a result. Since many of these questions remain unsolved, the need for continued research and study into this critical area of modern environmental risk assessment is also highlighted.

\section{References}

Armitage P, Doll R (1954) The age distribution of cancer and a multi-stage theory of carcinogenesis. Br J Cancer 8: 1-12

Crump KS (1984) A new method for determining allowable daily intake. Fundam Appl Toxicol 4: 854-871

U.S. EPA (2000) Benchmark Dose Technical Guidance Document. External Review Draft number EPA/630/R-00/001. U.S. Environmental Protection Agency, Washington, DC 